\title{
A fluence convolution method to account for respiratory motion in three-dimensional dose calculations of the liver: A Monte Carlo study
}

\author{
Indrin J. Chetty, ${ }^{\text {a) }}$ Mihaela Rosu, Neelam Tyagi, Lon H. Marsh, Daniel L. McShan, James \\ M. Balter, Benedick A. Fraass, and Randall K. Ten Haken \\ Department of Radiation Oncology, The University of Michigan, Ann Arbor, Michigan 48109-0010
}

(Received 18 February 2003; accepted for publication 22 April 2003; published 25 June 2003)

\begin{abstract}
We describe the implementation of a fluence convolution method to account for the influence of superior-inferior (SI) respiratory induced motion on a Monte Carlo-based dose calculation of a tumor located in the liver. This method involves convolving the static fluence map with a function describing the SI motion of the liver-the motion function has been previously derived from measurements of diaphragm movement observed under fluoroscopy. Significant differences are noted between fluence-convolved and static dose distributions in an example clinical treatment plan; hot and cold spots (on the order of 25\%) are observed in the fluence-convolved plan at the superior and inferior borders of the liver, respectively. This study illustrates that the fluence convolution method can be incorporated into Monte Carlo dose calculation algorithms to account for some of the effects of patient breathing during radiotherapy treatment planning, thus leading to more accurate dose calculations. (C) 2003 American Association of Physicists in Medicine.
\end{abstract}

[DOI: $10.1118 / 1.1581412]$

The standard computation of dose distributions in conformal radiotherapy is based on a single instance of patient anatomy. However, as a consequence of patient breathing and the associated changes in organ anatomy, differences will result between the planned dose distribution and that actually delivered. Therefore, the incorporation of uncertainties due to organ motion resulting from respiration is a very important requirement for accurate dose calculation within a given treatment plan. ${ }^{1-5}$ One particular method to account for respiratory-induced organ motion is to convolve the static dose distributions with functions that approximate the breathing. ${ }^{4,5}$ A study by Lujan et al. ${ }^{4}$ has shown that applying a single convolution to the static dose distribution, for patients undergoing irradiation of the liver, is sufficient to predict the dose distribution for the given treatment plan; intrafraction effects were found to average out over the course of many fractions. A potential limitation of the dose convolution approach is that it is based on the assumption that the dose distribution is spatially invariant, i.e., the convolution of dose is conducted in an assumed homogeneous medium without cognizance of the variation in patient tissue densities. In a recent study, Beckham et al., ${ }^{6}$ have applied a fluence-convolution method to incorporate random setup error in the dose calculation. Specifically, Beckham et al. ${ }^{6}$ use the Monte Carlo method to sample the photon fluence that is first convolved with a 2D Gaussian random setup error kernel. Beckham et al. ${ }^{6}$ point out that fluence convolution is more accurate than dose convolution in heterogeneous media since fluence convolution correctly models the spatial variation of dose resulting from movement due to setup error. In this study, we extend the concept of fluence convolution to account for respiratory motion in a clinically realistic treatment plan for a lesion located within the liver. We compare calculations using the fluence convolution method with those from the static (no motion) case, as well as with a treatment plan generated using the dose convolution method.
The treatment planning study has been conducted using the UMPlan (University of Michigan, Ann Arbor, MI) treatment planning software. Dose calculations for both fluence and dose-based convolution have been performed using the Dose Planning Method (DPM) Monte Carlo code system, ${ }^{7,8}$ which has been integrated within UMPlan. The Monte Carlo virtual source model used for patient-specific dose calculations is similar to that developed by Chetty et al., ${ }^{9}$ and is described at length in that paper. However, a brief description of the source model is necessary here. The treatment head components of a Varian 21EX linear accelerator (Varian Associates, Palo Alto, CA) were simulated using the BEAMnrc Monte Carlo code (CNRC, Ottawa, CN). A virtual source model was reconstructed from the phase space distribution to sample the source particle's position, energy, and direction. Arbitrary field shapes are simulated by multiplying the uncollimated fluence map by a matrix describing the multileaf collimator (MLC) configuration-the composite fluence matrix includes a correction for leaf edge penumbral effects. ${ }^{9}$

The fluence convolution method to correct for respiratoryinduced motion of the liver was implemented by convolving the static fluence distribution, $\Phi_{\text {static }}$, with a probability distribution function associated with breathing induced diaphragmatic displacement, labeled $F_{\text {motion }}$ in this paper, to generate a composite fluence map, $\Phi_{\text {motion }}$, which is used to sample the source particle's position. $\Phi_{\text {static }}$ represents the MLC-shaped field static fluence distribution and is located in a plane perpendicular to the beam central axis, between the accelerator and the patient. For a point, $r$, that includes the motion, we then have

$$
\begin{aligned}
\Phi_{\text {motion }}(r) & =F_{\text {motion }} \otimes \Phi_{\text {static }} \\
& =\int_{r^{\prime}} F_{\text {motion }}\left(r-r^{\prime}\right) \Phi_{\text {static }}\left(r^{\prime}\right) d r^{\prime} .
\end{aligned}
$$




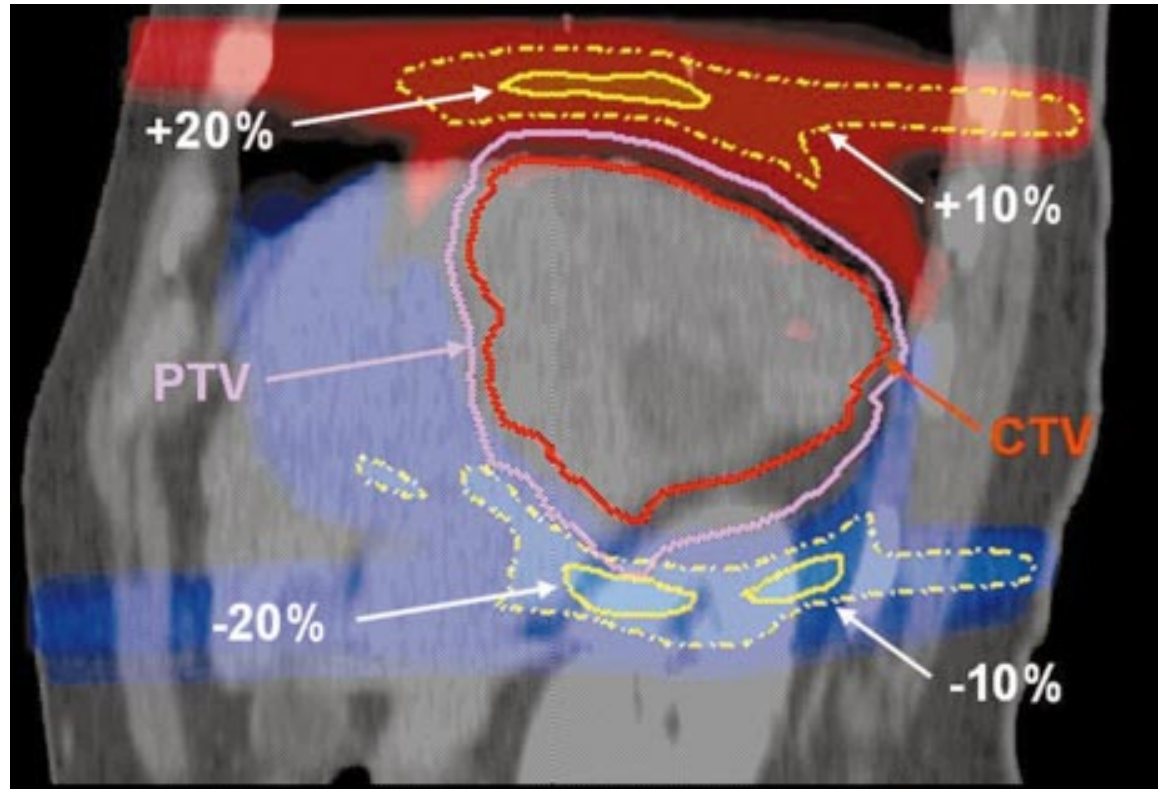

FIG. 1. Percentage dose difference map (fluence convolved dose-static dose) in the sagittal view. Both treatment plans have been normalized to $100 \%$ at the isocenter. Included are the isodose lines corresponding to the $\pm 20 \%$ differences (solid yellow lines) and the $\pm 10 \%$ differences (dashed yellow lines). Nonhighlighted differences (dark regions) are within $\pm 2 \%$.

$F_{\text {motion }}$ has been developed by Lujan et al., ${ }^{4}$ based on the correlation between the liver and the diaphragm movements (as observed under fluoroscopy by Balter et al. ${ }^{10}$ ) and is given by

$$
\begin{aligned}
F_{\text {motion }}(z)= & \left\{n a \pi\left(\frac{z_{0}-z}{a}\right)^{(2 n-1) / 2 n}\right. \\
& \left.\times\left[1-\left(\frac{z_{0}-z}{a}\right)^{1 / n}\right]^{1 / 2}\right\}^{-1} \\
& \text { for } z_{0}-a<z<z_{0},
\end{aligned}
$$

where $z_{0}$ is the position at exhale, $a$ the amplitude of motion (patient specific, $1.5 \mathrm{~cm}$ in our study), and $n=3$, a parameter that describes the general shape of the model. In the Monte Carlo implementation, $F_{\text {motion }}$ is divided into 15 equally spaced probability bins, from the position at exhale $\left(z_{0}\right)$ to the position at inhale $\left(z_{0}-a\right)$. The position $(x, y$ at a fixed $z$ location) and energy for each particle starting from the virtual source is determined by first sampling $\Phi_{\text {static }}$. The source particle's incident direction is determined from the position coordinates assuming that the particle originated from a point, ${ }^{9}$ i.e., $u=x / R, v=y / R, w=z / R$, where $R$ $=\sqrt{ } x^{2}+y^{2}+z^{2}$. To account for the motion in the SI direction, $F_{\text {motion }}$ is sampled to determine the positional translation, $\delta z$; the particle's $z$ coordinate is then translated according to the relation: $z^{\prime}=z+\delta z$. Note that the particle's $x$ and $y$ coordinates remain unchanged as $F_{\text {motion }}$ incorporates motion in only the $z$ (SI) dimension. The following relation may then be used to describe the coordinate transformation from the static fluence distribution $\Phi_{\text {static }}$, in the unprimed coordinates, to the motion convolved fluence, $\Phi_{\text {motion }}$, in the primed coordinates:

$$
x^{\prime}=x, \quad y^{\prime}=y, \quad \text { and } z^{\prime}=z+\delta z .
$$

The direction cosine vectors for each source particle are also appropriately modified as there is a change in $R$ from the translation of $z$,

$$
\begin{aligned}
& u=\frac{x^{\prime}}{R}, \quad v=\frac{y^{\prime}}{R}, \text { and } w=\frac{z^{\prime}}{R}, \\
& \text { where } R=\sqrt{\left(x^{\prime 2}+y^{\prime 2}+z^{\prime 2}\right)} .
\end{aligned}
$$

The fluence convolution method described here is different from that of Beckham et al., ${ }^{6}$ where the $x, y$ position coordinates of each source particle in the phase space file are resampled according to a $2 \mathrm{D}$ Gaussian random setup error kernel. In addition to differences in the positional sampling and in the treatment head description (phase space versus virtual source model), Beckham et al. ${ }^{6}$ do not recalculate the direction vectors for each translated source particle, as we do in our implementation.

The influence of respiratory motion on the dose distribution was also evaluated using a dose convolution method, ${ }^{4}$ performed by convolving the static dose distribution, $D_{\text {static }}$ (calculated using $\Phi_{\text {static }}$ ) with the function, $F_{\text {motion }}$, described above. The dose at a point $r$, that includes the motion, is calculated as follows:

$$
\begin{aligned}
D_{\text {motion }}(r) & =F_{\text {motion }} \otimes D_{\text {static }} \\
& =\int_{r^{\prime}} F_{\text {motion }}\left(r-r^{\prime}\right) D_{\text {static }}\left(r^{\prime}\right) d r^{\prime},
\end{aligned}
$$

$F_{\text {motion }}$ is cast in the form of a $1 \mathrm{D}$ discrete matrix along the $z$ (SI) dimension of the patient, and is convolved with $D_{\text {static }}$ so that the integral shown in Eq. (1) is reduced to a summation.

The treatment plan beam configuration consisted of 15 MV anterior, lateral and oblique fields, combined with segmental fields (directed from the same angles) to produce a dose distribution of $100 \pm 5 \%$ within the planning target vol- 


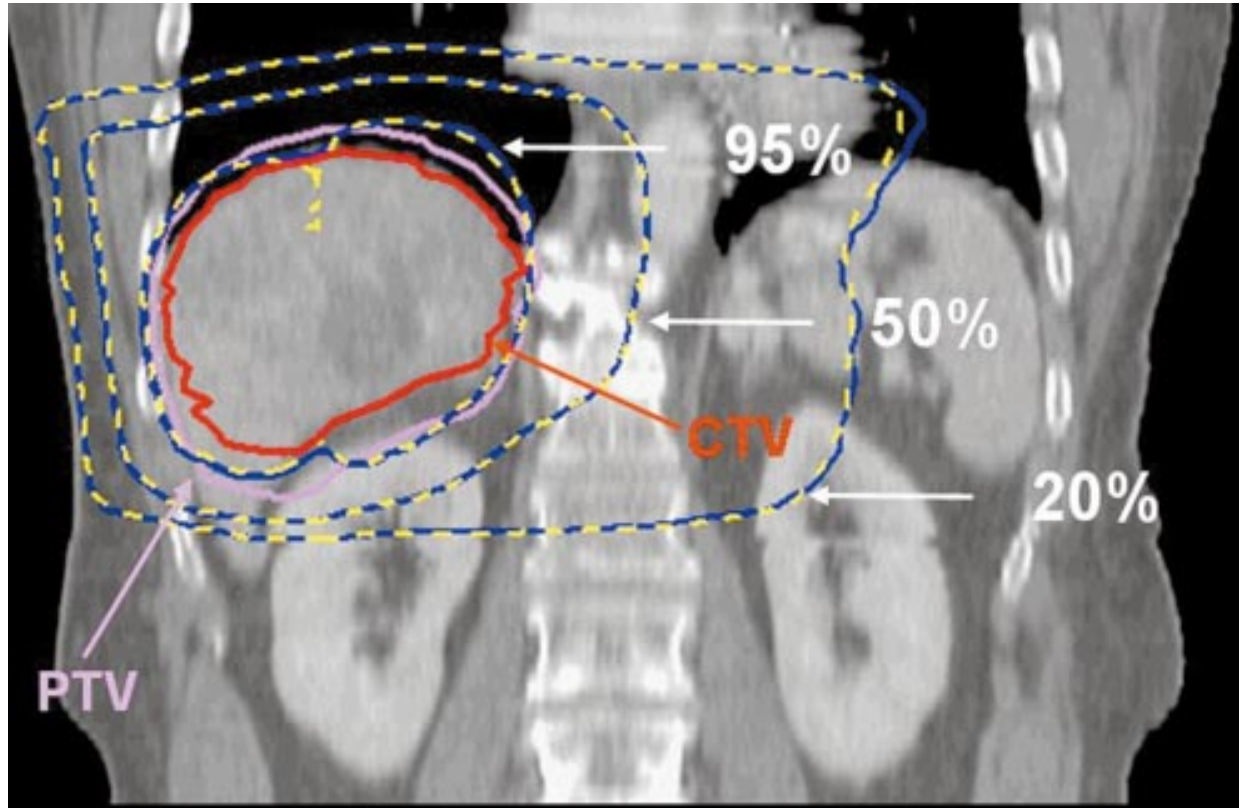

Fig. 2. Isodose lines: $95 \%, 50 \%$, and $20 \%$, calculated using the fluence convolution (solid blue lines) and dose convolution (dashed yellow lines) methods, in the coronal view. Both treatment plans have been normalized to $100 \%$ at the isocenter.

ume (PTV). The CT data set used for treatment planning was acquired at exhale, under voluntary breath hold. The treatment planning volumes: gross tumor volume (GTV), clinical target volume (CTV), and planning target volume (PTV) are those defined by the ICRU Report No. $50,{ }^{11}$ and are derived in this work from our current liver treatment protocol. ${ }^{12}$ The CTV is formed by a uniform, $1 \mathrm{~cm}$ expansion of the GTV.
The PTV includes a uniform $0.5 \mathrm{~cm}$ margin for setup uncertainties, as well as a $0.3 \mathrm{~cm}$ margin superiorly (added to account for the reproducibility of the exhale CT scan) and a $1.5 \mathrm{~cm}$ margin inferiorly to account for patient breathingthese expansions are performed relative to the CTV. ${ }^{4}$

DPM calculations were conducted using a voxel size of $3 \times 3 \times 1 \mathrm{~mm}^{3}$ (in the $x, y, z$ dimensions), a $2 \mathrm{~mm}$ step size,

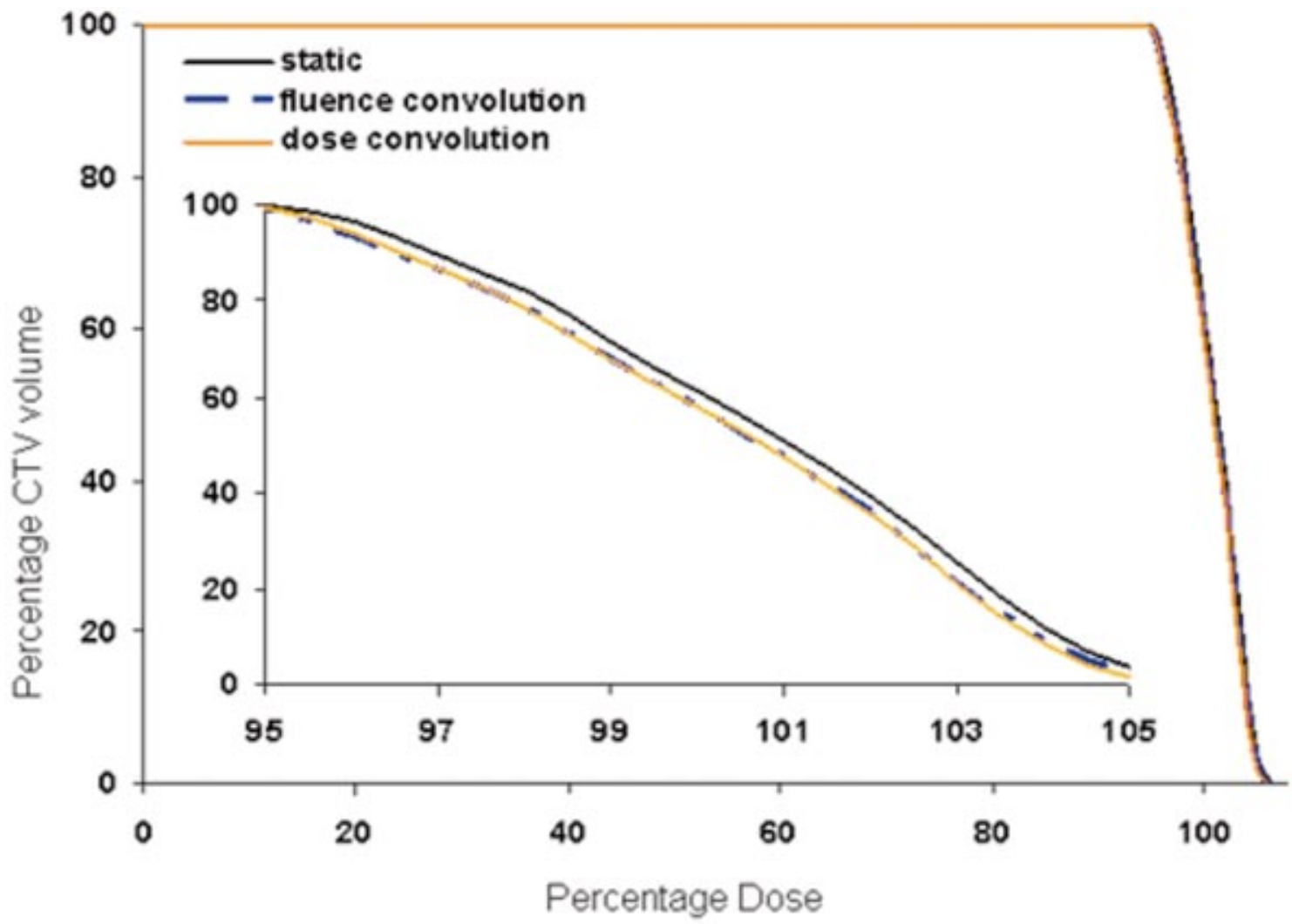

FIG. 3. Dose volume histograms for the CTV shown for the static (solid black line), fluence convolved (dashed blue line) and dose convolved (solid orange line) treatment plans. The inset represents a magnification of the high gradient region of the DVH, between the $95 \%$ and $105 \%$ dose values. 


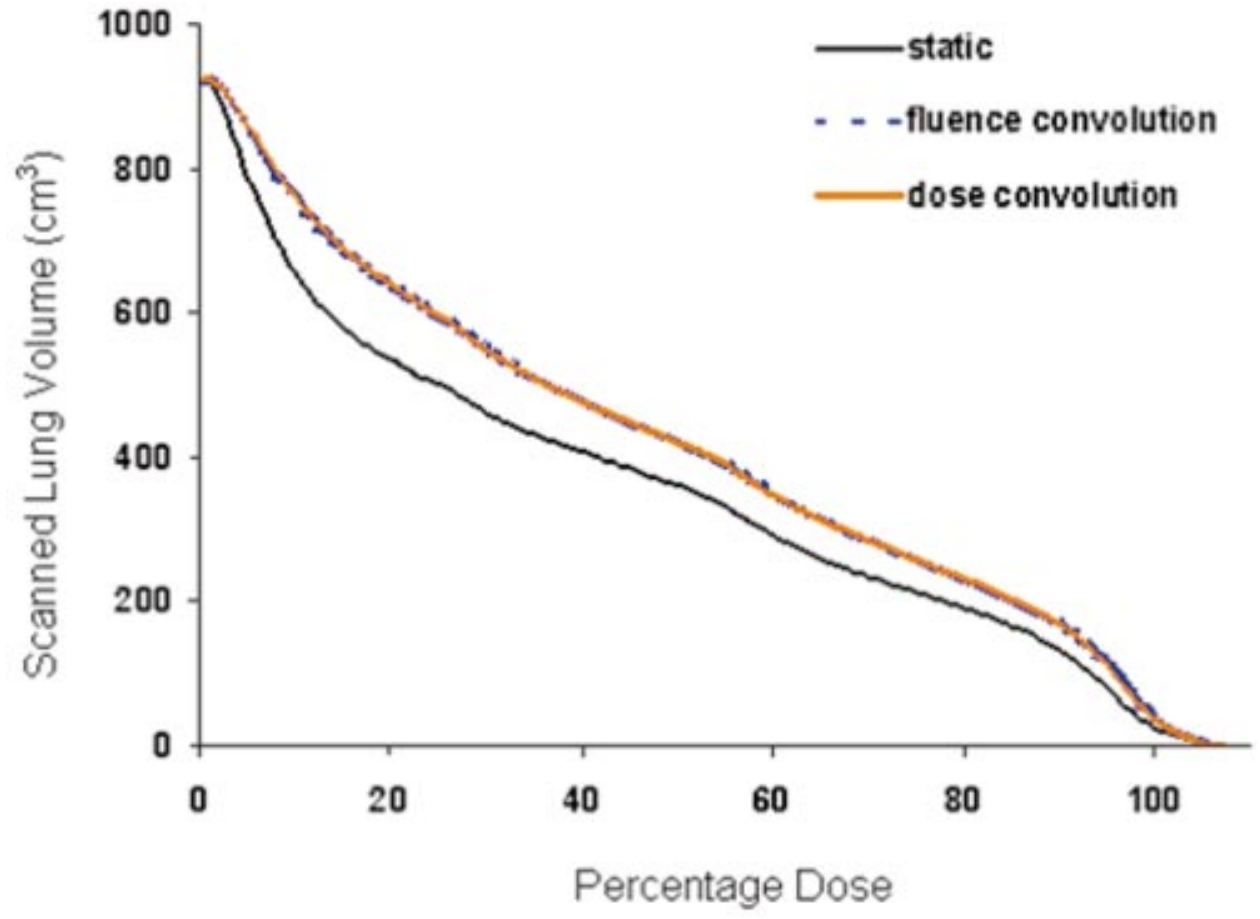

FIG. 4. Dose volume histograms for the CT-scanned right lung volume shown for the static (solid black line), fluence convolved (dashed blue line) and dose convolved (solid orange line) treatment plans.

and low energy electron and photon cutoff values of 200 and $50 \mathrm{keV}$, respectively. For each treatment plan, 6 billion histories were simulated ( 1 billion histories per field), resulting in $1 \sigma$ statistics of roughly less than $1.5 \%$ in calculated dose, if we combine the inherent uncertainty in the reconstructed fluence (from the virtual source) of $1 \%$. The time required for these simulations was approximately 10 hours per 1 billion particles, running on a single $1 \mathrm{GHz}$, VMS-based, Alpha processor.

Figure 1 illustrates a percentage difference map (in the sagittal view) between fluence convolved and static dose distributions. The difference map was calculated by subtracting the static dose from the fluence convolved dose for each point in the dose distribution. The fluence convolved and static treatment plans were normalized to $100 \%$ at the isocenter (located within the PTV), as is routinely done for patients planned on our liver treatment protocol. ${ }^{4,12}$ The difference between the absolute doses in the static and dose convolved plans at the isocenter, located within the central homogeneous region of the PTV, is negligible. The red color wash regions in Fig. 1 represent areas of positive dose differences indicating that the dose in the fluence convolved plan is higher than that in the static plan. Similarly, the blue color wash corresponds to regions where the fluence convolved doses are lower than those in the static treatment plan. Also, presented in Fig. 1 are the isodose regions corresponding to differences of $\pm 20 \%$ (in the solid lines) and $\pm 10 \%$ (in the dashed lines). Maximum point differences of $+25 \%$ are observed in the region superior to the PTV, located within the lung. The differences observed in Fig. 1 are a consequence of the motion of the liver due to breathing. Given that the CT scan was acquired at the exhale position, it is expected that, during inhale, the expansion of the lung will force the liver to move inferiorly. This means that the region of lung tissue located superiorly to the PTV will be exposed to radiation, while the inferior portion of the PTV will be blocked and will tend to be under-dosed, as noted in Fig. 1. Presented in Fig. 2 are the 95\%, 50\%, and 20\% isodose lines for the treatment plans generated using the fluence convolution (solid blue lines) and the dose convolution (dashed yellow lines) methods, in the coronal view. The differences between these two plans are within $\pm 2 \%$ on average, however, the differences might well be larger had the PTV not extended outside the liver into the lung; the influence of the PTV, protruding into the lung, tends to minimize differences between the spatially invariant dose distribution (an assumption required for dose convolution) and the spatially variant dose distribution (as calculated with fluence convolution). The reason for this is that the Monte Carlo static dose calculation is expected to correctly account for the spread of dose into the lung (at the superior edge of the PTV); convolving the static dose in this region is therefore likely to be as accurate as the fluence convolved calculation. It is clear, however, that a much more extensive investigation (involving many treatment plans) is necessary before conclusions can be drawn regarding the dosimetric differences between the fluence and dose convolution approaches used in accounting for breathing-induced organ motion in the vicinity of the liver.

Analyses of dose volume histograms (DVHs) for the CTV and the right lung were conducted for the static, fluence and dose convolved treatment plans. For the CTV, DVH differences between the static and the motion convolved (fluence or dose convolved) dose distributions are relatively small (as noted in Fig. 3) indicating that the margin between CTV and PTV adequately accounts for the motion. However, in this particular example, differences in the PTV DVHs between the static and motion convolved doses were also found to be negligible. A likely reason for this is that only the very su- 
perior and inferior regions of the PTV are influenced by the motion, so that the resulting influence on the DVH is small. Figure 4 illustrates DVHs for the scanned right lung volume generated from the static and motion convolved treatment plans. Significant differences are noted for the motionconvolved plans in comparison to the static treatment plan. For example, the volume of lung receiving doses greater than $50 \%$ of the isocenter dose is $17 \%$ higher in the fluence convolved treatment plan relative to the static case. Such differences may be clinically relevant.

In this study, we have implemented a fluence convolution method to account for the influence of liver motion (in the superior-inferior direction) due to breathing on the 3D dose distribution. We have tested this method on a clinical treatment plan and have found significant differences between the fluence convolved and static dose distributions, indicating the importance of organ motion in treatment planning. Although the fluence convolution method assumes a rigid-body approximation and ignores intrafraction effects much like the dose convolution approach, it can be incorporated to provide a more accurate dose distribution. The focus of future work will be to conduct an extensive treatment planning study of the fluence convolution method, involving lesions located at various locations within the liver as well as in other anatomical regions, such as the lung.

\section{ACKNOWLEDGMENTS}

This work has been supported in part by NIH Grant No. P01-CA59827 and by a University of Michigan Cancer Center grant funded by the John and Suzanne Munn Endowment.
${ }^{a)}$ Electronic mail: indrin@med.umich.edu

${ }^{1}$ G. S. Mageras et al., "A method of incorporating organ motion uncertainties into three dimensional conformal treatment plans," Int. J. Radiat. Oncol., Biol., Phys. 35, 335-342 (1996).

${ }^{2}$ M. A. Moerland et al., "The influence of respiration induced motion of the kidneys in the accuracy of radiotherapy treatment planning, a magnetic resonance imaging study," Radiother. Oncol. 30, 150-154 (1994).

${ }^{3}$ J. M. Balter et al., "Uncertainties in CT-based radiation therapy treatment planning associated with patient breathing," Int. J. Radiat. Oncol., Biol., Phys. 36, 167-174 (1996).

${ }^{4}$ A. E. Lujan et al., "A method for incorporating organ motion due to breathing into 3D dose calculations," Med. Phys. 26, 715-720 (1999).

${ }^{5}$ S. D. McCarter et al., "Evaluation of the validity of a convolution method for incorporating tumour movement and setup variations into the radiotherapy planning system," Phys. Med. Biol. 45, 923-931 (2000).

${ }^{6}$ W. A. Beckham et al., "A fluence-convolution method to calculate radiation therapy dose distributions that incorporate random set-up error," Phys. Med. Biol. 47, 715-720 (1999).

${ }^{7}$ J. Sempau et al., "DPM, a fast, accurate Monte Carlo code optimized for photon and electron radiotherapy treatment planning calculations," Phys. Med. Biol. 45, 2263-2291 (2000).

${ }^{8}$ I. J. Chetty et al., "Photon beam relative dose validation of the DPM Monte Carlo code in lung-equivalent media," Med. Phys. 30, 563-573 (2003).

${ }^{9}$ I. J. Chetty et al., "A virtual source model for Monte Carlo modeling of arbitrary intensity distributions," Med. Phys. 27, 166-172 (2000).

${ }^{10} \mathrm{~J}$. M. Balter et al., "Improvement of CT-based treatment planning models of abdominal targets using static exhale imaging," Int. J. Radiat. Oncol., Biol., Phys. 41, 939-943 (1998).

${ }^{11}$ ICRU Report 50. International Commission on Radiation Units and Measurements: Prescribing, recording and reporting photon beam therapy, Bethesda, Maryland, 1993.

${ }^{12}$ T. S. Lawrence et al., "Treatment of cancers involving the liver and porta hepatis with external-beam irradiation and intraarterial hepatic fluorodeoxiuridine,’ Int. J. Radiat. Oncol., Biol., Phys. 20, 555-561 (1991). 